\title{
Le indagini dirette a supporto della caratterizzazione dei siti contaminati
}

\author{
simone bisogno ${ }^{1}$ \\ ${ }^{1}$ Tecnologie per l'ambiente
}

June 30, 2020

\begin{abstract}
Le indagini ambientali sono un insieme di analisi effettuate sulle diverse matrici ambientali ossia acqua, aria e suolo appartenenti a un determinato luogo. L'obiettivo è quello di individuare un'eventuale contaminazione (superamento concentrazione soglia di contaminazione: CSC) e i conseguenti livelli di inquinamento della stessa, in modo da promuovere interventi di bonifica e riqualificazione ambientale. Le indagini possono essere condotte mediante due tipologie: dirette o indirette. Nel presente articolo vengono citati aspetti dell'indagine diretta le quali a loro volta si suddividono in due categorie: geognostiche e di caratterizzazione della contaminazione. A disciplinare le diverse indagini e le diverse procedure amministrative degli interventi di bonifica sono: l'All. 2, Titolo V, Parte IV del D. Lgs. 152/2006, il “Piano di indagini ambientali”' e il DM 471/99 che fornisce le informazioni riguardo la localizzazione dei punti di campionamento e il numero minimo di sondaggi da effettuare (sostituito dalla normativa vigente). Viene, inoltre, citato e mostrato un caso studio nel comune di Cernusco sul Naviglio (MI) riguardante la caratterizzazione dello stato chimico, morfologico ed ambientale dei terreni costituenti l'area in esame.
\end{abstract}

\section{Introduzione}

L'allegato 2 del D. Lgs. 152/2006 (G.U. Allegato 2 - Criteri generali per la caratterizzazione dei siti contaminati. 14/04/2006, n.d.) fornisce quelli che sono i criteri generali per la caratterizzazione dei siti contaminati. Le attività di caratterizzazione devono essere condotte in modo da permettere la validazione dei risultati finali da parte delle Pubbliche Autorità. Il piano di indagini dovrà contenere la dettagliata descrizione delle attività che saranno svolte in campo ed in laboratorio per la caratterizzazione ambientale del sito.

Le indagini avranno l'obiettivo di:

- verificare l'esistenza di inquinamento di suolo, sottosuolo e acque sotterranee; definire il grado, l'estensione volumetrica dell'inquinamento; delimitare il volume delle aree di interramento di rifiuti;

- individuare le possibili vie di dispersione e migrazione degli inquinanti dalle fonti verso i potenziali ricettori;

- ricostruire le caratteristiche geologiche ed idrogeologiche dell'area al fine di sviluppare il modello concettuale definitivo del sito;

- ottenere i parametri necessari a condurre nel dettaglio l'analisi di rischio sito specifica;

- individuare i possibili ricettori.

A tal fine, devono essere definiti:

- l'ubicazione e tipologia delle indagini da svolgere, sia di tipo diretto, quali sondaggi e piezometri, sia indiretto, come i rilievi geofisici; 
- il piano di campionamento di suolo, sottosuolo, rifiuti e acque sotterranee;

- il piano di analisi chimico-fisiche e le metodiche analitiche;

- la profondità da raggiungere con le perforazioni, assicurando la protezione degli acquiferi profondi ed evitando il rischio di contaminazione indotta dal campionamento;

- le metodologie di interpretazione e restituzione dei risultati.

Il D.Lgs 152/06 si limita a definire le strategie adottabili per selezionare l'ubicazione dei punti di sondaggio e prelievo delle matrici ambientali e non fornisce quindi alcuna indicazione circa la densità areale dei punti di campionamento.

Il Dm 471/99 ( ARPAT - Agenzia regionale per la protezione ambientale della Toscana -DM 25/10/99, $N^{\circ}$ 471 , n.d.) , invece, riportava il numero minimo di sondaggi da effettuare in funzione dell'estensione del sito da investigare e del tipo di matrice da campionare.

Si precisa che nei casi in esame successivi all'emanazione del D.Lgs. 152/06, il D.M. 471/99 è stato utilizzato solo per alcuni aspetti tecnici non specificati nel D.Lgs. 152/06 (punti di investigazione per suolo, sottosuolo e acque sotterranee sulla base delle dimensioni del sito).

Una sostanziale differenza tra i due decreti è costituita dalla definizione di sito contaminato, da cui ne consegue l'obbligatorietà dell'elaborazione dell'Analisi di Rischio per il calcolo delle CSR nell'attuale norma. Il D.Lgs. 152/06 introduce, inoltre, la definizione di sito potenzialmente contaminato, nel caso in cui si rileva un superamento delle CSC, rispetto al sito contaminato in cui si è verificato il superamento delle CSR. Pertanto il sito, ai sensi del D.Lgs. 152/06, definito come sito potenzialmente contaminato corrisponde al sito contaminato, definito dal D.M. 471/99. Il D.M. 471/99 imponeva infatti l'applicazione delle procedure di bonifica al superamento dei valori CSC tabellari.

In letteratura sono presenti numerosi manuali e testi che indicano metodologie e linee guide per lo svolgimento del piano di indagine. Tra queste vi è il manuale fornito dall'APAT (N. Calace, M. Fratini, M. Guerra, F. Pascarella, F. Zampetti. Manuale per le indagini ambientali nei siti contaminati. Planetearth. Manuele Apat 43/2006, n.d.) (Agenzia per la protezione dell'ambiente e per i servizi tecnici) con nome: 'Manuale per le indagini ambientali nei siti contaminati'. Quest'ultimo tratta i temi legati ai siti contaminati, in particolare le indagini da condurre per la determinazione delle caratteristiche delle matrici ambientali, con particolare riguardo al suolo, sottosuolo e alle acque sotterranee. 


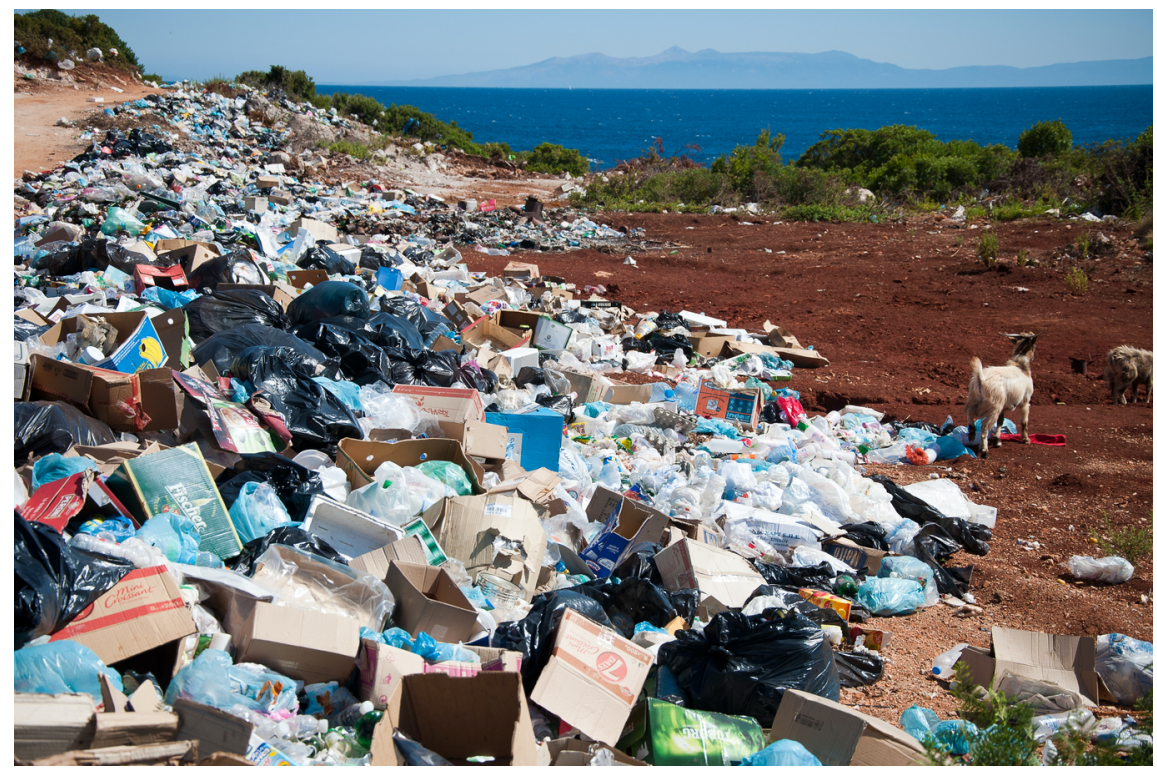

Figure 1: Discarica abusiva di rifiuti

\section{Le indagini dirette}

Il sottosuolo può essere caratterizzato dal punto di vista geologico, geotecnico e geomeccanico attraverso indagini:

- indirette che misurano la variazione di determinate caratteristiche del sottosuolo e permettono di risalire successivamente alla definizione del terreno che lo costituisce;

- dirette che consentono un rilievo diretto e dettagliato del terreno.

Le indagini dirette sono sempre necessarie e le indagini geofisiche (o indirette) non possono prescindere dai metodi diretti che si basano sull'analisi di campioni prelevati direttamente in situ.

$\mathrm{Ci}$ sono diverse strategie di campionamento in funzione delle necessità:

- prelievo ed analisi di campione medio;

- prelevo ed analisi di campioni per intervalli di profondità (ad esempio ogni metro);

- prelievo ed analisi di campioni per variazioni significative di litologia;

- prelievo ed analisi di campioni in relazione a caratteristiche organolettiche quali colore, odore, consistenza, etc.

Nel caso di indagine di suolo superficiale si ricorre a metodi di scavo meccanizzato o manuale tramite l'utilizzo di:

- utensili manuali (come palette), che consentono di prelevare direttamente il campione dalla superficie o dalle pareti di uno scavo eseguito con escavatore;

- carotieri manuali, ovvero trivelle che consentono di prelevare campioni direttamente fino ad una profondità di 1,5 metri circa;

- pale meccaniche, nel caso di campioni a profondità più elevate, fino a 4 metri.

Le indagini dirette si suddividono in due categorie, geognostiche e di caratterizzazione della contaminazione.

Le indagini geognostiche (F.Cestari . Indagini geognostiche in sito. Dario Flaccovio Editore. Maggio 2013, n.d.) sono indagini atte a conoscere e caratterizzare il sottosuolo. Tali indagini hanno quindi lo scopo di con- 
sentire la ricostruzione geolitostratigrafica delle formazioni incontrate e la loro parametrizzazione geotecnica. La scelta della metodologia d'indagine avviene in base alle normative generali e regionali, oltre che ai fattori legati al singolo sito, quali le dimensioni e l'accessibilità del sito, l'omogeneità delle matrici ambientali, la tipologia degli inquinanti e la loro distribuzione spaziale. Le tipologie d'indagini sono le seguenti:

- Prospezioni geofisiche

- Sondaggi e prove geotecniche in sito con prelievo di campioni di terreno

- Scavi superficiali (pozzetti) con prelievo di campioni di terreno e dell'acqua di falda, se presente

- Installazione di piezometri per il controllo della falda ed il prelievo periodico di campioni d'acqua

- Prove idrauliche per la determinazione delle caratteristiche dell'acquifero.

I metodi geofisici sono in grande evoluzione; allo stato attuale i più diffusi che forniscono informazioni non quantitative sulla presenza di inquinanti, sono:

- Georadar (Ground Penetrating Radar)

- Elettromagnetici

- Resistivi

- Sismici e rifrazioni

- Magnetometrici

Occorre evitare di mettere in contatto terreni e falde differenti e, pertanto, si deve ricorrere all'impiego di rivestimenti telescopici per isolare acquiferi e terreni. Una delle cose più importanti a cui bisogna porre maggiore attenzione è la sicurezza nelle indagini nei siti inquinati. In proposito si fa riferimento al Decreto del Ministero dell'Ambiente n. 471 del 25 Ottobre 1999.

La seconda tipologia di indagini dirette sono, come già citato, quelle di caratterizzazione della contaminazione, consistono in prove in sito o in laboratorio finalizzate alla valutazione dello stato di contaminazione delle varie matrici ambientali. L'obiettivo dell'operazione di campionamento del terreno, nell'ambito della caratterizzazione della contaminazione, consiste nel prelievo di un campione che sia il più rappresentativo possibile delle caratteristiche chimiche, fisiche, biologiche degli orizzonti attraversati ed indicatore dell'eventuale presenza di sostanze inquinanti. Dalle indagini dirette si possono trarre vantaggi e svantaggi. Tra i vantaggi è sicuramente opportuno evidenziare l'elevata precisione delle informazioni ottenute; al contrario invece con queste indagini si segue una stima puntuale della qualità delle matrici ambientale e vi è una difficile rappresentazione dell'eterogeneità del fenomeno di contaminazione.

Uno dei problemi riguardante questa tipologia di indagine è quello riferito alla gestione dei materiali da scavo, infatti le implicazioni di carattere ambientali ed economiche sono enormi. In sintesi sono tre i possibili scenari di gestione dei materiali da scavo:

- Sottoprodotti, impiego come TRS (terre e rocce da scavo)

- Non rifiuto (rinterro del sito)

- Rifiuto (Recupero / EoW : End of waste ) 


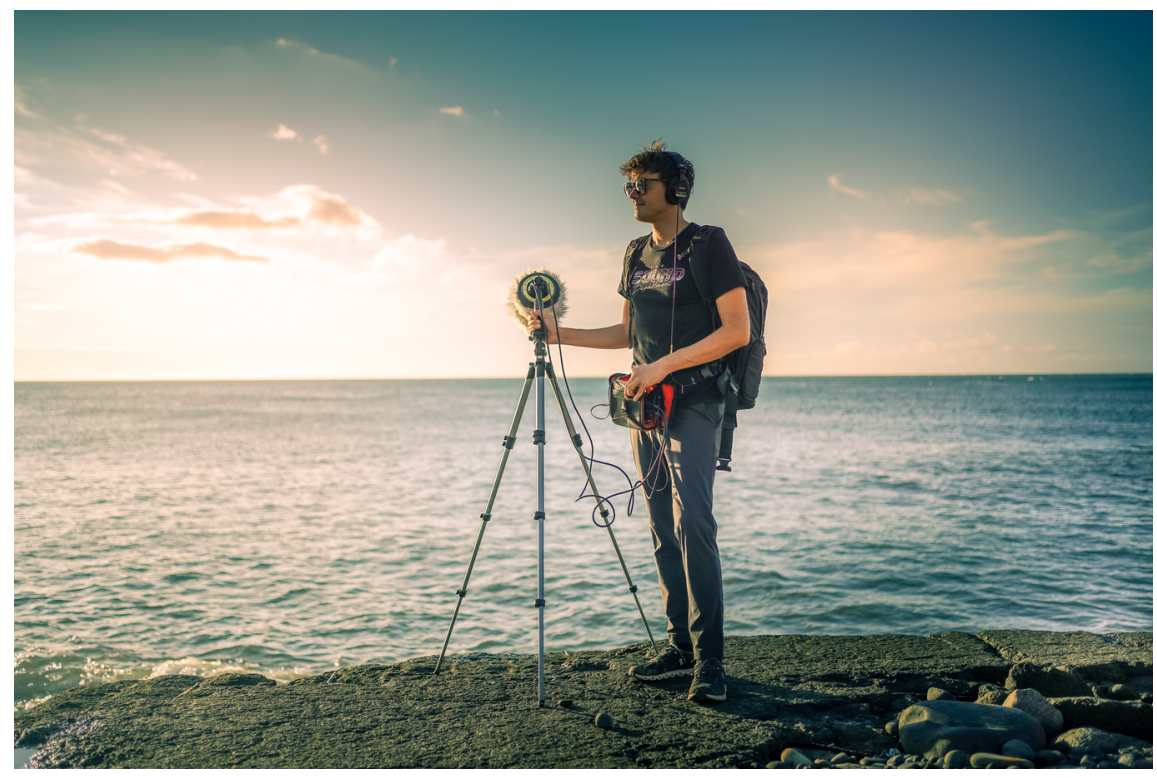

Figure 2: Indagini dirette

Un caso studio analizzato evidenzia come l'obiettivo principale quello di poter fornire una caratterizzazione esaustiva dello stato chimico, morfologico ed ambientale dei terreni costituenti l'area in esame ( $D r$. Geol. C. Leoni. Piano indagini ambientali. 05/03/2014, n.d.) . L'area oggetto di indagine si colloca all'interno di un contesto rurale / residenziale. La medesima risulta storicamente interessata dalla sola presenza di un prato stabile occasionalmente sfalciato. Dopo aver condotto diversi sopralluoghi all'interno dell'area è stato possibile affermare che, nella stessa, non è stata rilevata la presenza di pozzi perdenti né di serbatoi interrati o di qualsiasi altra attività antropica in grado di impattare negativamente sul suolo e sul primo sottosuolo e quindi escludere la presenza di potenziali sorgenti di contaminazione del suolo e del primo sottosuolo. In particolare in corrispondenza dell'area in esame la falda è situata ad una profondità di circa $11 \div 13 \mathrm{~m}$. da p.c. Tenuto conto delle caratteristiche strutturali dell'area di indagine, delle attività pregresse condotte all'interno della stessa, delle previsioni di utilizzo della medesima e delle potenziali fonti di inquinamento del suolo presenti, sono stati realizzati 4 punti di indagine per il prelievo di campioni di terreno. La scelta e la localizzazione dei punti di campionamento si è basata sull' esame dei dati storici e sulla identificazione delle aree maggiormente vulnerabili nei confronti di una possibile contaminazione dei suoli in funzione della tipologia di attività svoltasi.Si specifica come non siano stati ubicati punti di indagine in corrispondenza delle serre per non arrecare danni alle colture in corso né, tantomeno, all'interno del casolare in muratura per non arrecare danni strutturali all'edificio stesso (il quale, comunque, non risulta interessato da alcuna possibile attività antropica in grado di impattare negativamente sul suolo e sul primo sottosuolo in posto).Tutti i punti di indagine sono stati predisposti attraverso l'esecuzione di trincee esplorative eseguite mediante l'impiego di un escavatore a braccio rovescio e realizzate in modo da consentire la descrizione ed il campionamento del profilo stratigrafico verticale fino ad una profondità pari a circa 2,00 metri dal piano di imposta della trincea stessa. Le analisi chimiche effettuate sui campioni di terreno hanno interessato le sostanze che potenzialmente potrebbero essere presenti nel sito di indagine, in relazione alla storia pregressa dello stesso. Tutti i campioni sono stati sottoposti a vagliatura diretta in sito con un setaccio avente maglie di $2 \mathrm{~cm}$ prima di essere sistemati in appositi barattoli di vetro con chiusura ermetica. Il set analitico individuato per ciascun campione acquisito viene brevemente riportato di seguito:

- Metalli pesanti (As, Cd, CrTOT, Cr6, Hg, Ni, Pb, Cu, Zn)

- Idrocarburi $(\mathrm{C}>12, \mathrm{C}<12)$

I valori riscontrati attraverso le analisi chimiche effettuate sui campioni di terreno prelevati nell'ambito della 
campagna d'indagine, sono stati comparati con i valori di concentrazione limite accettabili per i suoli ad uso verde pubblico, privato e residenziale. In ordine cronologico sono state eseguite le seguenti operazioni:

- Descrizione dell'area in esame e attività produttive pregresse (inquadramento corografico e riferimenti catastali, attività pregresse e centri di pericolo, documentazione fotografica)

- Inquadramento geologico ed idrogeologico

- Caratterizzazione dei terreni (Punti di indagine, modalità di campionamento, analisi di laboratorio e risultati)

- Risultanze dell'indagine preliminare

\section{Conclusione}

Come si è visto, la programmazione delle indagini è una parte importante del complesso di attività di progettazione, costruzione e soprattutto per la caratterizzazione ambientale del sito. La programmazione infatti deve essere mirata in funzione della complessità del progetto, delle caratteristiche dell'area da indagare e delle diverse situazioni al contorno. Le indagini dirette, pertanto, rappresentano uno strumento fondamentale per verificare la presenza e l'individuazione di un'eventuale contaminazione e uno strumento atto a prevenire, impedire ed eliminare la diffusione di sostanze inquinanti al suolo e alle acque sotterranee non contaminati. Nel caso studio in tutti i campioni esaminati non è stato evidenziato il superamento della CSC imposte dalla vigente normativa e quindi è stato possibile attestare lo stato di salubrità del suolo.

\section{References}

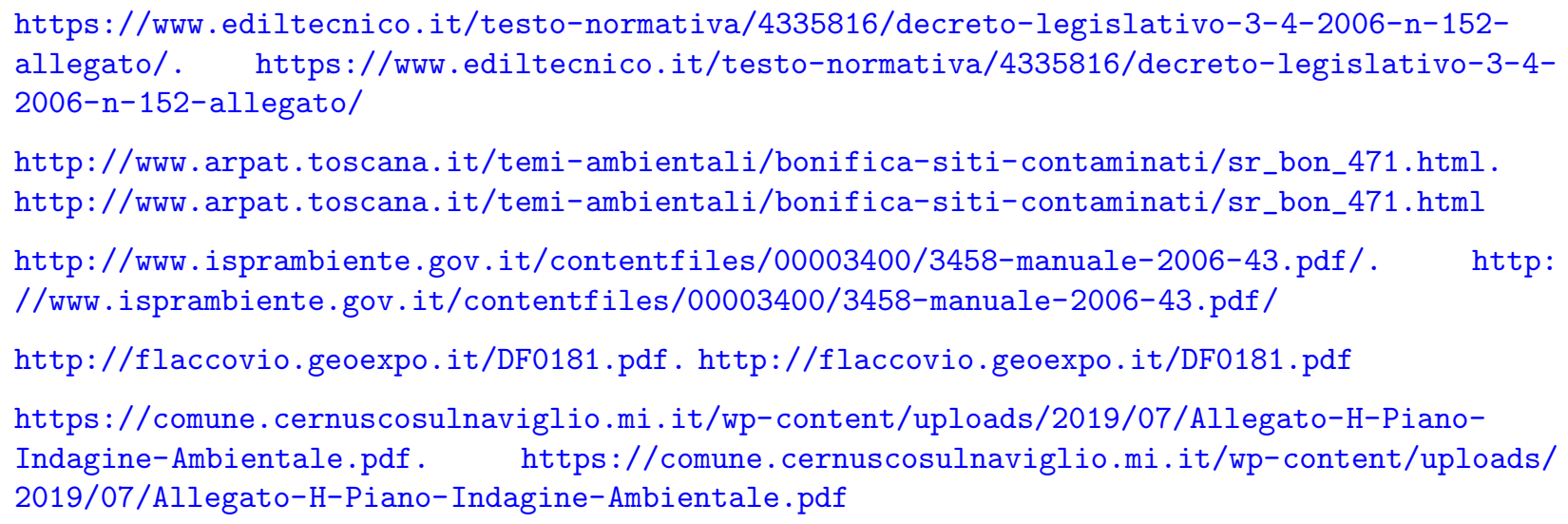

